\title{
Exotic (non-SUSY) Searches at the LHC
}

\author{
Ping Tan (on behalf of the ATLAS/CMS Collaborations) \\ Fermilab \\ P.O. Box 500, Batavia, IL 60510 - USA
}

\begin{abstract}
New studies on exotic (non-SUSY) searches beyond the Standard Model are reviewed, which include contact interactions using jet $p_{T}$ spectrum, leptoquark production, signals for new gauge bosons and extra dimensions, monojet events expected in ADD extra dimension scenarios, 4th generation quarks, and heavy stable charged particles [1]. Emphasis is given to the potential for early discoveries at the LHC, e.g., within the first year of physics data taking.
\end{abstract}

\section{Introduction}

Despite its amazing agreement with experimental data, there are theoretical indications and experimental evidence suggesting that the SM is only a low energy effective theory of a more fundamental theory. There are many intriguing questions we could ask ourselves about the SM. Are the quarks or leptons fundamental particles? Is the SM gauge symmetry a subset of an extended gauge symmetry? What is the nature of the electro-weak symmetry breaking (EWSB)? Almost every aspect of the SM has been extended to shed light on what could be the beyond-the-SM (BSM) theory. The extension of the SM gauge symmetry group has led the grand unification theory (GUT) of the electro, weak, and strong forces [2]. Several other GUT-inspired extensions were also discussed in the literature [3] [4]. So far the Higgs boson predicted in the SM has not been found experimentally. In addition to its existence, the Higgs mass suffers quadratic divergence due to the radiative corrections at one-loop level, the hierarchy problem. A possible solution to this problem is the Supersymmetry (SUSY). Other alternatives were also proposed, such as the little Higgs Model [5], Extra Dimensions [6] [7], Technicolor and extended Technicolor Models [8]. Many of these phenomenological models predict possible new physics at a $\mathrm{TeV}$ scale, which could be extensively explored at the LHC [9] $]^{\text {a }}$ Both ATLAS [10] and CMS [11] have a $4 \pi$ general-purpose hadron-collider detector, which is suitable for high- $p_{T}$ physics studies at the LHC.

\section{New Gauge Bosons}

Experimentally the most studied Z' bosons are the ones predicted in the Sequential Standard Model (SSM), the Left-Right Symmetry Model (LRSM), and the E6 Model $\left(Z_{\chi}^{\prime}, Z_{\psi}^{\prime}, Z_{\eta}^{\prime}\right)$ [4]. Direct searches for these gauge bosons at Tevatron set limits on the $Z^{\prime}$ mass from several hundreds $\mathrm{GeV}$ up to about $1 \mathrm{TeV}$ [12] [13] [14]. Indirect constraints from electro-weak global analysis have also been undertaken by the LEP experiments [15]. The experimental signature of Z' bosons is a narrow resonance on top of the Drell-Yan mass spectrum. Both ATLAS [16] and CMS [17] [18] studied experimental sensitivities for the above models in both $Z^{\prime} \rightarrow e^{+} e^{-}$and $Z^{\prime} \rightarrow \mu^{+} \mu^{-}$decay channels. The overall trigger efficiency for signal events are over $90 \%$ for both CMS and ATLAS. The major background is the irreducible Drell-Yan

${ }^{a}$ Note that all studies discussed in this paper assume a $14 \mathrm{TeV}$ center-of-mass energy.

DIS 2009 


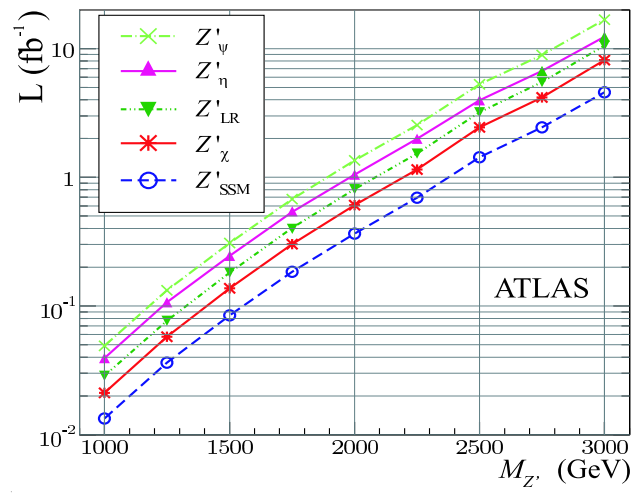

Figure 1: The required luminosity for $5 \sigma$ discovery as a function of the Z' mass. production. Others, such as QCD multijet events, $\mathrm{W} / \mathrm{Z}+$ jets, SM diboson production, and $t \bar{t}$ also contribute. Figure 1 shows the required luminosity needed for a $5 \sigma$ discovery of Z' $\rightarrow e^{+} e^{-}$as a function of Z' mass. With less than $100 \mathrm{pb}^{-1}$ of integrated luminosity, sensitivity beyond the Tevatron experiments could be achieved. The Z' $\rightarrow$ $\mu^{+} \mu^{-}$channel has very similar sensitivity. The CMS studies resulted in similar sensitivities as well.

Both ATLAS and CMS also studied experimental sensitivities to search for a W' bosons in the so-called extended SM model [19]. The leptonic final states, $W^{\prime} \rightarrow$ $\mu \nu$ and $W^{\prime} \rightarrow e \nu$, were used in the study.

The neutrino in the final state escapes detection and can only be inferred by the Missing Transverse Energy (MET). The experimental signature is a high- $p_{T}$ lepton accompanied by large MET. The major background is QCD multijet events, $\mathrm{W} / \mathrm{Z}+$ jets, and $t \bar{t}$ production. Both ATLAS [20] and CMS [21] studies obtained similar sensitivities. With about $100 \mathrm{pb}^{-1}$ of luminosity, discovery is possible up to the W' mass of about $2.2 \mathrm{TeV}$. Current best experimental limit on the $\mathrm{W}^{\prime}$ boson is $m_{W^{\prime}}>1 \mathrm{TeV}$ at $95 \%$ C.L. from the $\mathrm{D} 0$ experiment [22].

\section{Extra Dimensions and Black Holes}

The Arkani-Hamed, Dimopoulos, Dvali (ADD) model was suggested to solve the hierarchy problem between the electro-weak scale and the Planck scale by introducing a number $\delta$ of Extra Dimensions (ED) [6]. In the simplest scenario all the EDs are compactified over a torus and have the same radius $R$. The fundamental scale $M_{D}$ is related to the $M_{\text {planck }}$, $M_{\text {planck }}^{2}=8 \pi M_{D}^{\delta+2} R^{\delta}$. If the scale $M_{D}$ is of the order of TeV, light Kaluza-Klein gravitons can be directly produced at LHC, $q \bar{q} \rightarrow g G, g g \rightarrow g G$. Gravitons interact weakly with ordinary matter, therefore they escape detection and can only be inferred from the MET. The most stringent limits on the scale $M_{D}$ range from $0.83 \mathrm{TeV}$ up to $1.6 \mathrm{TeV}$ at $95 \%$ C.L, depending on the number of EDs [23] [24]. CMS conducted a search for EDs with jet + MET final states. With $100 \mathrm{pb}^{-1}, 3 \sigma$ evidence could be achieved for $M_{D}$ of $3.58 \mathrm{TeV}(2.62$ $\mathrm{TeV}$ ) for $\delta=2(4)$. In the absence of EDs, one could push the current experimental limits on the $M_{D}$ for $\delta=2$ or 4 by a factor of three higher [25].

If the scale $M_{D}$ is low enough, Black Holes (BHs) could be produced at LHC [26]. BHs decay to all SM particles and have event signature of high multiplicity and spherical event shape. ATLAS conducted a search for BHs. In this study, the classical BH cross-section at parton level is given by $\hat{\sigma}_{q q^{\prime} \rightarrow B H}=\pi r_{h}^{2}$, where $r_{h}$ is the so-called horizon radius [27]. The production cross section for a $\mathrm{BH}$ with mass range between $5-14 \mathrm{TeV}$ is at the order of 10 $\mathrm{pb}$. The scaler summation of the $p_{T}$ of each object in the event was used to distinguish the $\mathrm{BH}$ events from SM processes. A BH with mass $>5 \mathrm{TeV}$ threshold could be discovered with only a few $\mathrm{pb}^{-1}$ of integrated luminosity [28].

DIS 2009 


\section{Alternatives to Electro-Weak Symmetry Breaking Mechanism}

Strongly interacting theories, such as the Technicolor and the extended Technicolor, provide a dynamical solution to the EWSB. ATLAS performed a search for the techni-rho $\left(\rho_{T C}\right)$ and techni-omega $\left(\omega_{T C}\right)$ proposed in Technicolor Strawman Model [29] via the dimuon final states. This process has the same final states as the $Z^{\prime}$ decays and similar analysis techniques were used. The study showed that with less than $5 \mathrm{fb}^{-1}$ of integrated luminosity, the $\rho_{T C}$ and $\omega_{T C}$ with mass up to $1 \mathrm{TeV}$ can be discovered [16]. The current best limits on these technifermions were given by the CDF collaboration [30].

In the absence of a light Higgs boson, the mechanism of EWSB will be best studied in the processes of vector boson scattering (VBS). Several models predicted the presence of new vector or scalar boson resonances at $\mathrm{TeV}$ scale [31]. The Chiral Lagrangian model with Pade unitarisation provides a framework to study the VBS at high mass. ATLAS has performed a search for these vector and scalar WW, WZ, or ZZ resonances via lepton+2jet+MET, or dilepton+dijet, or dilepton+MET final states. The discovery of resonances in VBS at high mass will take a few tens of $\mathrm{fb}^{-1}[32]$.

\section{4th-generation, Excited Quarks, and Lepto-Quarks}

A 4th-generation of massive fermions with the same quantum numbers as the known SM fermions has been actively searched for in high-energy experiments. The direct searches for the 4th-generation $t^{\prime}$ set the limit $m_{t^{\prime}}>311 \mathrm{GeV}$ at $95 \%$ C.L. by the CDF experiment [33]. The experimental sensitivity to search for a $b^{\prime}$ has studied by the CMS experiment. In this study, it was assumed that the mass of the $b^{\prime}$ is above the threshold of $b^{\prime} \rightarrow t W$ decay, therefore the dominated process is $p p \rightarrow b^{\prime} \bar{b}^{\prime} \rightarrow t \bar{t} W^{+} W^{-}$. With $100 \mathrm{pb}^{-1}$ of luminosity, more than $5 \sigma$ significance can be achieved for a $b^{\prime}$ of mass $300 \mathrm{GeV}$. With the same luminosity, in the absence of $b^{\prime}$ signal events we can set the mass limit of the $b^{\prime}$ up to $450 \mathrm{GeV}$ at $95 \%$ C.L., as shown in Fig. 2 [34].

In addition to searches for 4th-generation, CMS also conducted an analysis to search for excited quarks. In quark compositeness quarks can be excited to higher energy state by absorbing gluons and subsequently decay into quark-gluon final state, $q g \rightarrow q^{*} \rightarrow q g$. The dijet invariant mass spectrum were used to extract the possible signal. Figure 2 shows the fractional difference of different excited quarks from the purely QCD predictions of dijet mass spectrum. With less than $10 \mathrm{pb}^{-1}$ of luminosity, a $2 \mathrm{TeV}$ excited quark could be discovered [35]. The current limit on the excited quark is $>0.87 \mathrm{TeV}$ at $95 \%$ C.L. from the D0 experiment [36].

Leptoquarks (LQ) are hypothetical particles motived to explain the apparent symmetries between leptons and quarks [37]. The LQs carry both quark and lepton quantum numbers. There are experimental limits favoring three generation of LQs, therefore each LQ decays to a lepton and a quark from the same generation. At LHC, LQs can be produced either strongly or in association with a lepton via the LQ-quark-lepton coupling. The pair production cross section of LQs at LHC, $p p \rightarrow L Q L Q$, is about $2 \mathrm{pb}$ for a LQ with mass of $400 \mathrm{GeV}$ [38]. ATLAS studied the experimental sensitivities for the first and second generation LQs in the dilepton + dijet final state. With less than $100 \mathrm{pb}^{-1}$ of luminosity, both 1st and 2nd generation scalar LQ pair production could be discovered, provided that the mass of the LQs is smaller than $500 \mathrm{GeV}$ [39]. Experimental limits on the LQs are about 200-300 GeV at $95 \%$ C.L., depending on the branching fraction of the $L Q \rightarrow l q$ decay [40].

DIS 2009 

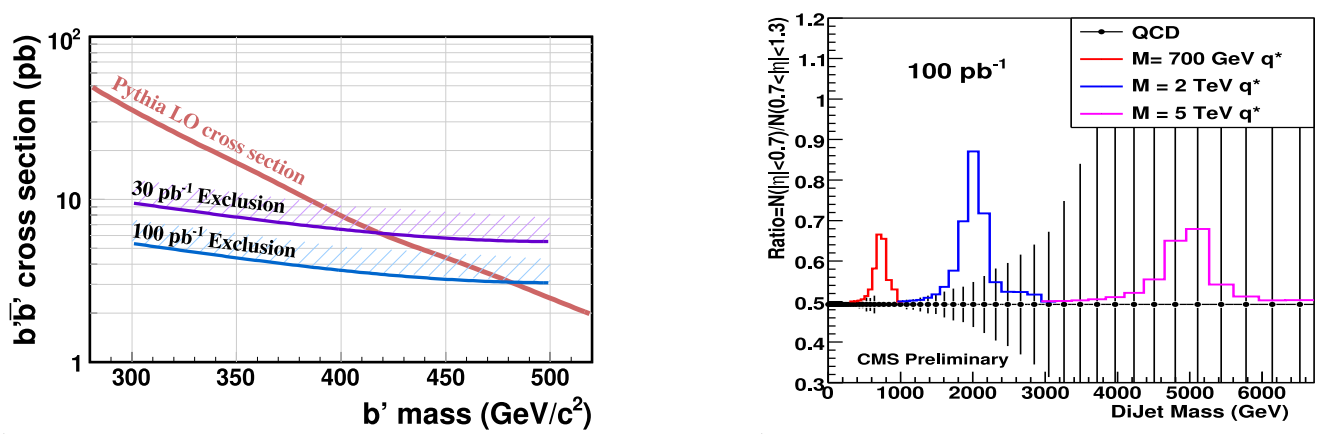

Figure 2: Left: the exclusion limits at the $95 \%$ C.L. on the $p p \rightarrow b^{\prime} \bar{b}^{\prime}$ production. Right: dijet resonance production compared to that in QCD.

\section{Contact Interactions}

New physics like quark compositeness or new interaction at a high scale $(\Lambda)$ can be modeled as a contact interaction at much lower dijet mass or jet $p_{T}$. Such interactions can manifest as a large rate of jet production in the high- $p_{T}$ region. CMS studied the sensitivity to contact interactions using the leading jet $p_{T}$ spectrum [35]. The study showed that with less than $10 \mathrm{pb}^{-1}$ of luminosity, CMS has access to $\Lambda$ above the current experiment limit $\Lambda>2.7$ $\mathrm{TeV}$ at $95 \%$ C.L. from the D0 experiment [41].

\section{Heavy Stable Charged Particles}

Heavy Stable Charged Particles (HSCPs) are predicted in many BSM physics models. Such particles can be distinguished from SM particles by exploiting their unique signature: a low velocity, $\beta$, associated with a high momentum of order of a few hundreds GeV. CMS studied the physics potential of searching for HSCPs using stau in Gauge Mediated Supersymmetric Breaking, KK-states in ED, gluino in split-SUSY, and supersymmetric top as benchmarks. The $d E / d x$ in the CMS tracking system and the timing information in the CMS Barrel muon system were both used to reconstruct the $\beta^{-1}$. The study showed that HSCPs can be discovered with early data for different models and in different mass regions. The Stable gluino search with $1 \mathrm{fb}^{-1}$ is sensitive to gluino masses above $1 \mathrm{TeV}$ and the GMSB scenarios with stable stau can be discovered with a few $\mathrm{pb}^{-1}$ [42].

\section{Summary}

In summary, there is a very rich and exciting exotic physics program at LHC probing many fundamental aspects of the nature. With as little as $100 \mathrm{pb}^{-1}$ of integrated luminosity at $14 \mathrm{TeV}$ we could largely extend current experimental limits in searches for new gauge symmetry groups (W'/Z' bosons), Extra Dimensions, 4th generations, excited quarks, leptoquarks, etc. Nature could come up with a surprise and new physics at $\mathrm{TeV}$ scale could be discovered with early LHC data. 


\section{References}

[1] Slides: http: //indico. cern . ch/contributionDisplay . py? contribId=45\&sessionId $=2 \&$ conf $I d=53294$

[2] H. Georgi and S.L. Glashow, Phys. Rev. Lett. 32438 (1974).

[3] R.W. Robinett and J.L. Rosner, Phys. Rev. D26 2396 (1982).

[4] M. Cvetic, P. Langacker, and B. Kayser, Phys. Rev. Lett. 68 (1992).

[5] N. Arkani-Hamed, A.G. Cohen, and H. Georgi, Phys. Lett. B513 232 (2001).

[6] N. Arkani-Hamed, S. Dimopoulos, and G.R.Dvali, Phys. Lett. B429 263 (1998).

[7] L. Randall and R. Sundrum, Phys. Rev. Lett. 833370 (1999).

[8] L.Susskind, Phys. Rev. D20 2619 (1979); E. Eichten and K.D. Lane, Phys. Lett. B90 125 (1980).

[9] L. Evans et al., JINST 3 S08001 (2008).

[10] ATLAS Collaboration, JINST 3 S08003 (2008).

[11] CMS Collaboration, JINST 3 S08004 (2008).

[12] Do Collaboration, V.M. Abazov et al., Phys. Rev. Lett. 95091801 (2005).

[13] CDF Collaboration, A. Abulencia et al., Phys. Rev. Lett. 96211801 (2006).

[14] CDF Collaboration, CDF/PUB/EXOTIC/PUBLIC/9160 (2008).

[15] Particle Data Group Collaboration, W.M. Yao et al., J. Phys. G33 (2006).

[16] ATLAS Collaboration, CERN OPEN-2008-020, 1696-1725 (2008).

[17] CMS Collaboration, CMS-PAS-EXO-08-001 (2008).

[18] CMS Collaboration, CMS-PAS-SBM-07-002 (2007).

[19] G. Altarelli et al., Z. Phys. C45 109 (1989).

[20] ATLAS Collaboration, CERN OPEN-2008-020, 1726-1749 (2008)

[21] CMS Collaboration, CMS-PAS-EXO-08-004 (2008).

[22] Do Collaboration, V.M. Abazov et al., Phys. Rev. Lett. 100031804 (2008).

[23] C. Ask, Proceedings of 32nd International Conference on High Energy Physics (ICHEP 2004), Vol. 2, 1289 (2005).

[24] CDF Collaboration, T. Aaltonen et al., Phys. Rev. Lett. 101181602 (2008).

[25] CMS Collaboration, CMS-PAS-EXO-08-011 (2008).

[26] S.B. Giddings and S.D. Thomas, Phys. Rev. D65 056010 (2002); S. Dimopoulos and G.L. Landsberg, Phys. Rev. Lett. 87161602 (2001).

[27] R.C. Myers and M.J. Perry, Ann. Phys. 172304 (1986).

[28] ATLAS Collaboration, CERN OPEN-2008-020, 1803-1827 (2008).

[29] K.D. Lane, Phys. Rev. D60 075007 (1999); K.D. Lane and S. Mrenna, Phys. Rev. D67 115011 (2003).

[30] CDF Collaboration, A. Abulencia et al., Phys. Rev. Lett. 95252001 (2005).

[31] M.S. Chanowitz, Presented at the 23rd International Conference on High Energy Physics, Berkeley, California, Jul 16-23, 1986.

[32] ATLAS Collaboration, CERN OPEN-2008-020, 1769-1802 (2008)

[33] CDF Collaboration, CDF public note-9446 (2008).

[34] CMS Collaboration, CMS-PAS-EXO-08-009 (2008).

[35] CMS Collaboration, CMS-PAS-SBM-07-002 (2007).

[36] Do Collaboration, V.M. Abazov et al., Phys. Rev. D69 111101 (2004).

[37] J.C. Pati, and A. Salam, Phys. Rev. D10 2751974.

[38] M. Kramer et al., Phys. Rev. D71 057503 (2005).

[39] ATLAS Collaboration, CERN OPEN-2008-020, 1750-1768 (2008)

[40] Do Collaboration, V.M. Abazov et al., Phys. Rev. Lett. 101241802 (2008); V.M. Abazov et al., Phys. Lett. B668 357 (2008); V.M. Abazov et al., Phys. Lett. B671 224 (2009).

[41] Do Collaboration, B. Abbott et al., Phys. Rev. Lett. 822457 (1999).

[42] CMS Collaboration, CMS-PAS-EXO-08-003 (2008).

DIS 2009 